\title{
Specimen Dependent Milling Directions for Correct Phase Identification in Multilayerd Thin Films
}

\author{
G.B. Thompson, R. Banerjee, and H.L. Fraser \\ Department of Materials Science and Engineering, The Ohio State University, 2041 College Road, \\ Columbus, Ohio 43210
}

Considerable debate has been generated on proper phase stability identification using electron beam diffraction for thin film multilayers [1-4]. This has been particularly relevant to the $h c p-f c c$ transitions reported in Ti/fcc metal multilayered thin films. It has been shown that hydrogen can artificially stabilize the $f c c$ Ti phase. Unfortunately, the 'hydrogen-problem' has prevented the understanding for which conditions $f c c$ Ti can be stabilized by a reduction in the interfacial free energy associated with the multilayered stack. In this proceeding, we will address the criteria necessary for proper phase identifaction in multilayered thin films by electron diffraction.

Transmission Electron Microscopy (TEM) foils have been prepared in both the plan-view and the cross-section geometry for two sets of Ti/Al multilayers [2]. The Ti/Al multilayer \#1 has a bilayer repeat thickness of $5.5 \mathrm{~nm} \mathrm{Ti} / 13.5 \mathrm{~nm} \mathrm{Al}$ where as Ti/Al \#2 has a bilayer repeat thickness of $1.6 \mathrm{~nm}$ $\mathrm{Ti} / 6.5 \mathrm{~nm} \mathrm{Al}$. As can be seen in Fig. 1, the plan-view electron diffraction pattern for Ti/Al \#1 was indexed as $h c p \mathrm{Ti} / f c c \mathrm{Al}$ where as the cross-section pattern was indexed as $f c c \mathrm{Ti} / f c c \mathrm{Al}$. The $f c c \mathrm{Ti}$ lattice parameter, in the cross-section, was $\approx 4.40 \AA$; this is a consistent lattice parameter for the $f c c \delta$ $\mathrm{TiH}_{2}$ phase. Contrary to this specimen, Fig. 2's diffraction pattern for Ti/Al \#2 was consistently indexed as $f c c \mathrm{Ti} / f c c \mathrm{Al}$ in both diffraction orientations with an $f c c$ Ti lattice parameter of $4.02 \AA$. This lattice parameter matches well with the predicted $f c c$ Ti lattice parameter by Paxton et al. [5].

The inconsistency of phase identification between the plan-view and cross-section TEM foils for the $\mathrm{Ti} / \mathrm{Al}$ multilayers can be understood in terms of the multilayer's orientation to the $\mathrm{Ar}^{+}$milling source during electron transparency thinning. In plan-view milling, the outer most layer can be a diffusion barrier to hydrogen into the multilayer stack as illustrated in Fig. 3(a). For Ti/Al multilayers, the diffusivity of hydrogen in $\mathrm{Al}$ is much less then that of Ti [6]. Even if the outer most layer of the multilayer is Ti, that Ti surface layer will be $f c c$ hydrogen stabilized, and the Al layer underneath this surface layer can prevent further diffusion of hydrogen into the multilayer. In the cross-section geometry, as shown in Fig. 3(b), the entire stack of freshly milled layers is exposed to the surface. In this orientation, the Al layer can not act as a diffusion barrier to hydrogen resulting in all the Ti layers being exposed to potential hydrogen stabilized $f c c$ phase. This simple diffusion cap model explains the discrepancies between the plan-view and cross-section diffraction patterns.

\section{References:}

[1] D. van Heerden, D. Josell, and D. Shechtman Acta Mater. 44(1) (1996) 297.

[2] R. Banerjee, S.A. Dregia, and H.L. Fraser Acta Mater. 47(15) (1999) 4225

[3] J. Bonevich and D. Josell Phys. Rev. Lett. 82(9) (1999) 2002.

[4] D. Josell, D. Shechtman, D. van Heerden Materials Letters 22 (1995) 275.

[5] A.T. Paxton, M. Methfessel, and H.M. Polatoglon Phys. Rev. B 41 (1990) 8127.

[6] E.A. Brandes and G.B. Brook, editors Smithells Metals Reference Book $7^{\text {th }}$ edt. ButterworthHeinemann London (1992). 


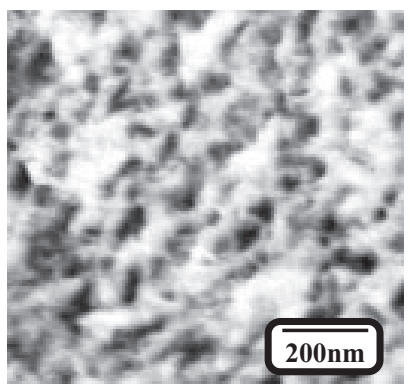

(a)

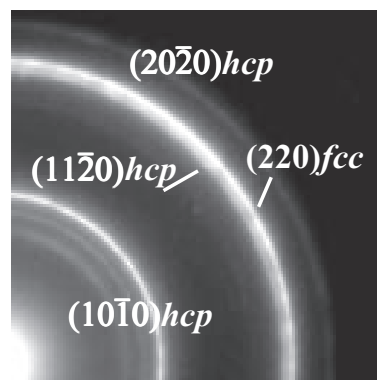

(b)

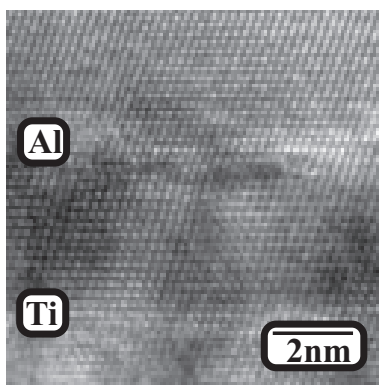

(c)

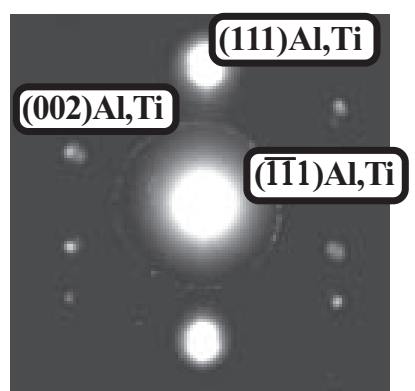

(d)

Fig. 1 Ti/Al \#1 Multilayer [2] (a) Plan-view bright-field many-beam image of the polycrystalline surface of the multilayer. (b) Plan-view diffraction pattern consistently indexed as $h c p \mathrm{Ti} / f c c \mathrm{Al}$.(c) High-resolution TEM of the multilayer cross-section of the film. (d) Cross-section diffraction pattern consistently indexed as $f c c \mathrm{Ti}(\mathrm{H}) / f c c \mathrm{Al}$.

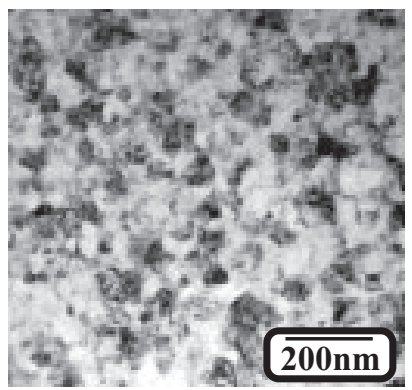

(a)

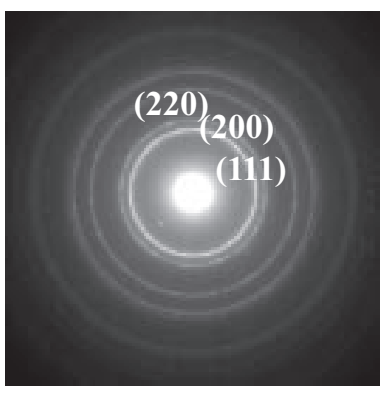

(b)

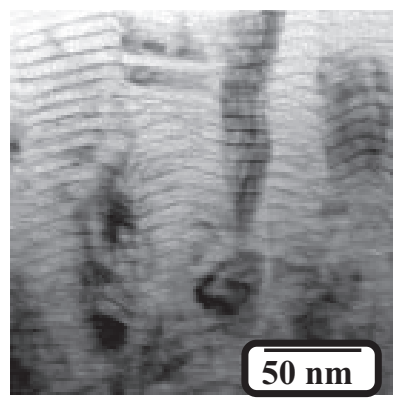

(c)

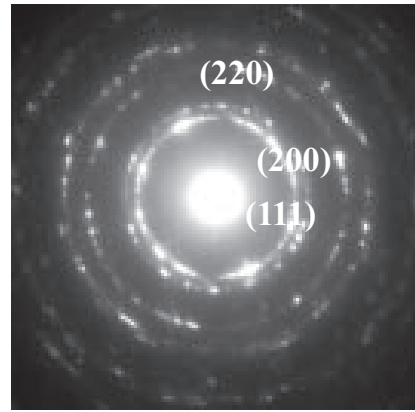

(d)

Fig. 2 Ti/Al \#2 Multilayer [2] (a) Plan-view bright-field many-beam image of the polycrystalline surface of the multilayer. (b) Plan-view diffraction pattern consistently indexed as $f c c \mathrm{Ti} / f c c$ Al.(c) Cross-section bright-field many-beam image of the multilayer. (d) Diffraction pattern of the cross-section indexed as $f c c \mathrm{Ti} / f c c \mathrm{Al}$.
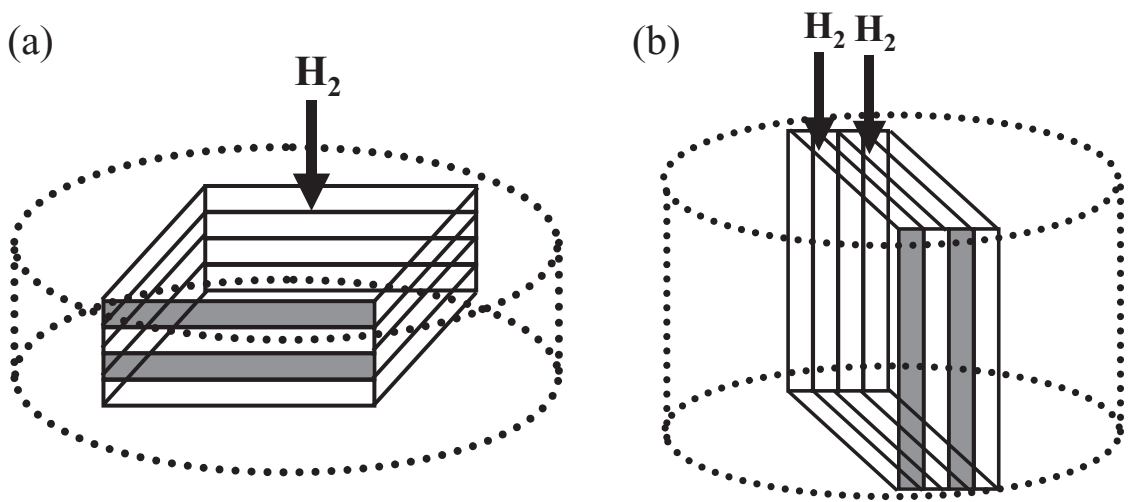

Fig. 3 Hydrogen contamination dependent milling directions (a) In the plan-view milling of the foil, the outer layer can act as a diffusion barrier to contmaintation throughout the stack. (b) In the crosssection milling direction, the freshly milled layers are exposed to the surface and potential contamination can result. 